\title{
¿Por qué (también) es tan difícil legislar sobre juego en España? Un 'déjà vu' de lo ocurrido con el alcohol
}

\author{
Why is it (also) so difficult to legislate gambling in Spain? \\ 'Déjà vu' of what occurred with alcohol
}

\author{
Mariano Chóliz*; Jerónimo Sáiz-Ruiz**. \\ *Facultad de Psicología, Universidad de Valencia. Consejo Asesor de juego Responsable, Dirección General de Ordenación \\ de Juego; ** Hospital Ramón y Cajal. Universidad de Alcalá. CIBERSAM, IRYCIS. Consejo Asesor de Juego Responsable, \\ Dirección General de Ordenación de Juego.
}

$\mathrm{H}$ ace unos años la revista Adicciones titulaba uno de sus editoriales “ ¿Por qué es tan difícil legislar sobre alcohol en España?” (Rodríguez-Martos, 2007) denunciando lo incomprensible que resulta el no regular adecuadamente para prevenir el alcoholismo en nuestro país, especialmente después de que se vieran los efectos positivos que tuvo para la salud pública la legislación sobre el tabaco. Hoy día, en lo que se refiere a la adicción al juego, nos encontramos en una situación muy parecida a la de entonces con el alcohol, aunque quizá más grave, ya que las recientes medidas regulatorias del juego han supuesto la promoción de una actividad que es la principal causa de la ludopatía, considerada como un trastorno mental tanto por parte de la Organización Mundial de la Salud (OMS) como por la Asociación Psiquiátrica Americana (APA). Desde los ámbitos científicos y clínicos se entiende que se trata de un trastorno de naturaleza adictiva y así fue recogido en la última edición del manual de diagnóstico DSM-5 (APA, 2013).

A pesar de la gravedad del problema y de la relación directa que existe entre disponibilidad y accesibilidad de los juegos, las sucesivas regulaciones sobre esta materia en nuestro país han ido incrementando exponencialmente la oferta y el acceso a los juegos, así como el atractivo y publicidad de los mismos. En el momento actual nos encontramos en una situación de absoluta permisividad respecto del juego de azar, pareciendo que las autoridades no son conscientes de los riesgos que tiene la promoción excesiva del juego de azar para la salud y el bienestar de los ciudadanos y de que las medidas regulatorias son la mejor fórmula para prevenir la adicción al juego.

La historia de la legislación sobre el juego de azar en España es realmente destacable. Contamos con uno de los reglamentos más antiguos del mundo en materia de regulación de la actividad de juego, como es El Ordenamiento de Tafurerías de Alfonso X el Sabio, que se trataba en realidad de un código mediante el cual se disponían de una serie de medidas para castigar los comportamientos deshonestos en el juego, con penas acordes a la Castilla de la Baja Edad Media. Seis siglos más tarde, en 1812 y en plena Guerra contra el Francés, se instaura la Lotería Nacional como una forma de recaudación de impuestos, al modo de lo que ya hiciera Carlos III varias décadas antes con la Lotería Napolitana, la cual puede considerarse como la antecesora de la actual Lotería Primitiva. Esto coincide con un punto de inflexión en lo que a regulación del juego se refiere, porque a partir del siglo XIX el juego de azar deja de tener una naturaleza esencialmente privada (es decir, entre los propios jugadores), para convertirse en una actividad económica de primera magnitud en dos relevantes dimensiones. Por un lado, como forma de recaudación de impuestos para el Estado, mediante loterías y otros sorteos de menor entidad. Por otro, como actividad lucrativa a través de la cual algunas empresas promueven negocios cuyos beneficios empresariales se basan en lo que juegan (y pierden) los jugadores. Nacen los casinos y salones de juego 
vinculados, principalmente, a ambientes sociales económicamente favorecidos, en los que apuestan las clases dominantes. A partir de entonces, y en la medida en que se trata de una actividad económica cuya regulación depende del Estado, el juego de azar que no estaba expresamente permitido, se consideraba prohibido. Dicha restricción alcanzó las principales cotas de prohibición durante la dictadura del general Franco — según algunos debido al rechazo que en el dictador había producido el que su padre hubiera sido jugador-, periodo durante el cual el juego de azar se consideraba un vicio.

En nuestra reciente historia como sociedad democrática han ocurrido dos hitos muy relevantes en lo que se refiere a la legislación del juego y en las implicaciones que dichas regulaciones han tenido respecto a la ludopatía. Se trata del Real Decreto Ley 16/1977 y la más reciente Ley 13/2011 de Regulación del Juego, a la que nos referiremos posteriormente.

El juego de azar fue completamente legalizado en España en 1977. Como acabamos de comentar, durante el franquismo gran parte del juego estaba prohibido y solamente estaban permitidas la Lotería Nacional, los sorteos de la ONCE, las quinielas deportivas y algunas apuestas minoritarias (galgos y caballos, principalmente). Después de la muerte de Franco se legalizó el juego como una forma de atracción de capital del turismo extranjero y de captación del nacional, que se iba a apostar a los países vecinos, principalmente a los casinos de Francia. Se legalizaron bingos, casinos y máquinas de apuestas "recreativas con premio" (las conocidas como "tragaperras") y se liberalizó el sector, permitiendo que el juego de azar fuera una actividad empresarial. Se abrieron salas de bingo, casinos y, muy singularmente, se instalaron máquinas en bares y restaurantes. A partir de ese momento, y en lo que se refiere a la adicción, las máquinas se convirtieron en España en las responsables de más del $80 \%$ de los casos de ludopatía.

Con independencia de las características estructurales de las máquinas, que las hacen potencialmente más adictivas que cualquier otro tipo de juego debido a la inmediatez de la recompensa, a que se basan en programas de reforzamiento de razón variable o a que inducen sesgos cognitivos que favorecen el seguir jugando a pesar de las recurrentes pérdidas, dos han sido las particularidades, ajenas a la propia máquina pero directamente relacionadas con los aspectos regulatorios, que todavía han potenciado más sus efectos adictivos. Tales son su enorme disponibilidad y fácil accesibilidad.

En lo que se refiere a la disponibilidad, desde que se legalizó el juego las máquinas fueron implantadas en bares y restaurantes de toda España y aunque, en honor a la verdad, no es algo que sea exclusivo de España, no es lo habitual en la mayoría de países, con el agravante de que los bares son un elemento sociológicamente central en nuestra sociedad. La proliferación de máquinas en tantos establecimientos de hostelería se explica por las serias deficiencias en materia de regulación del juego por parte del Ministerio del Interior de la UCD, así como por la concesión de licencias descontrolada durante los primeros gobiernos del PSOE, situación ésta que fue aprovechada -incluso incitada - por algunas empresas para convertirse en los auténticos dueños del sector de las máquinas, prácticamente en régimen de oligopolio. En la actualidad, contamos con más de doscientas mil de ellas, que son muchas más de las que hay en Las Vegas, distribuidas a lo largo de toda la geografía española en la mayoría de los bares. Además, es preciso tener en cuenta que en el bar se suele beber alcohol y que éste dificulta el necesario control conductual que precisa el jugador para no verse inmerso en una vorágine de juego y de las consiguientes pérdidas que todo juego de azar provoca cuando se apuesta con excesiva frecuencia.

En cuanto a la accesibilidad, a diferencia de bingos y casinos, en los cuales es preciso identificarse para entrar en los salones, el acceso al bar es libre, sin necesidad de tener que acreditarse ni de ningún otro requisito especial que sea necesario para poder jugar. Además, el mecanismo de juego es simple, puesto que la mayor complicación que se exige con estas máquinas es la de introducir una moneda por una ranura y accionar una palanca o botón. Tan sencillo como barato, puesto que con veinte céntimos ya se puede jugar.

Es decir, en España cualquier persona tiene en su entorno más cercano muchas máquinas —que son los juegos más adictivos-, en lugares donde se suele beber alcohol -que dificulta el control y favorece conductas de riesgo-, ante las cuales no necesita ningún tipo de acreditación que pueda limitar el juego excesivo. Como consecuencia de ello, hasta la llegada del juego online el cuarenta por ciento del dinero gastado en todos los juegos legales de azar se juega en estas máquinas; en 2015 fueron casi diez mil millones de euros. Moneda a moneda.

El quid de la cuestión es que tanto la disponibilidad como la accesibilidad, que —al igual que ocurre con el alcohol y otras drogas- son dos de los principales factores implicados en el desarrollo de la adicción, pueden ser controladas con adecuados sistemas de regulación; es decir, con leyes y reglamentos apropiados que tuvieran como uno de sus principales objetivos la prevención de los trastornos adictivos. Ése es, como ocurre con el alcohol, uno de los principales retos que tiene la legislación española actualmente en materia de juego.

\section{Iniciativas de regulación}

El desarrollo de las tecnologías de la comunicación e información (TIC) también ha supuesto una revolución en el juego de azar. En la actualidad, los juegos son mu- 
cho más accesibles gracias a los dispositivos electrónicos, al tiempo que la conexión a Internet ha supuesto el desarrollo de nuevas modalidades de juego y un incremento en la oferta, es decir, una mayor disponibilidad. En este campo el legislador llegó tarde, hasta el punto que cuando se propuso legalizar el juego online mediante la Ley 13/2011 de Regulación del Juego, hacía ya mucho tiempo que las empresas de juego online estaban operando en España de forma ilegal, o alegal (puesto que no tenían autorización para dicha actividad), aunque no clandestina, puesto que durante años han sido visibles con el patrocinio de equipos de la Liga de Fútbol Profesional. Se daba la paradoja de que la publicidad de juego estaba prohibida para casinos, bingos y máquinas, cuya actividad estaba regulada y era legal, mientras que las empresas de apuestas deportivas o póquer online no solamente ejercían su actividad de esta forma anómala, sino que tenían una enorme presencia mediática.

En las motivaciones de la Ley 13/2011 de Regulación del Juego se encontraban, entre otras, la prevención de la ludopatía y, a tal efecto, se creó el Consejo Asesor de Juego Responsable, un órgano consultivo cuyas decisiones no son vinculantes para el Gobierno y cuyos principales objetivos eran el proponer una Estrategia de Juego Responsable y el asesoramiento sobre cuestiones referidas a la puesta en marcha de la Ley que tuvieran que ver con la promoción de hábitos saludables de juego.

En lo que se refiere a la Estrategia, los miembros del Consejo Asesor que suscriben estas líneas propusieron un modelo de regulación que fue presentado tanto en el Congreso de los Diputados como en la propia Dirección General de Ordenación del Juego (DGOJ), pero dichas recomendaciones no fueron atendidas por parte del Ejecutivo y tampoco fueron bien aceptadas por parte de las empresas del sector que forman parte de dicho Consejo Asesor. Algunos de los aspectos sustanciales de esta propuesta aparecen en el artículo de esta misma revista: "Regular el juego para prevenir la adicción: hoy más necesario que nunca”(Chóliz y Sáiz, 2016).

Otras recomendaciones que propusimos en materia de regulación del juego, como son la prohibición de las estrategias de marketing que inducen un juego excesivo (por ejemplo los bonos gratuitos para jugar) o de las slot online ("tragaperras" online), tampoco se tuvieron en cuenta. De hecho, los bonos, que inicialmente se utilizaban solamente en póquer online, también se han extendido a otro tipo de apuestas, mientras que las slot online son el tipo de juego online que está experimentando un mayor crecimiento en lo que se refiere al gasto. Según los datos de la DGOJ, sólo en el primer trimestre de 2016 los españoles gastaron más de 247 millones de euros en este tipo de máquinas de apuestas (DGOJ, 2016a). Hemos pasado de tener dos máquinas tragaperras en cada bar a disponer de ellas en cada uno de nuestros móviles.
En su lugar, las estrategias de juego responsable aplicadas por las empresas consisten simplemente en una serie de recomendaciones genéricas, presentadas como si fueran las condiciones legales de la letra pequeña de un contrato, a las que se accede rastreando por la web tras sortear todo tipo de banners, anuncios o ventanas emergentes de publicidad y estrategias de marketing incitadoras al juego e irresistibles para el jugador patológico. Entre los sellos de calidad, otorgados por asociaciones privadas que no tienen por qué estar relacionados con la promoción de la salud se encuentra el del propio Ministerio, denominado "Juego Seguro”. Se trata de un logo equívoco, ya que da una falsa sensación de seguridad al jugador al ser respaldado por la propia Administración, pero lo único que quiere decir es que la empresa está autorizada a operar. Es decir, más que "Juego Seguro" quiere decir "Juego Legal".

También es cuestionable la propia página de juego responsable de la DGOJ, denominada "JugarBien.es". Estando de acuerdo en que la información sobre prevención es necesaria, la Administración no puede quedarse en este punto y no tomar las necesarias medidas de prevención basadas en la regulación, máxime cuando la información sobre el juego se presenta de forma naif, minimizando los riesgos del juego y las consecuencias de la ludopatía. No obstante, lo más cuestionable de esta iniciativa es la atribución de la responsabilidad de la enfermedad al propio jugador por el hecho de no jugar bien, cuando lo que es cierto es que las condiciones ambientales incitan a un juego excesivo y el jugador patológico, por definición, es incapaz de dejar de jugar. Y las condiciones ambientales, como estamos viendo, se regulan mediante la legislación correspondiente.

Pero en lo que se refiere a la legislación de juego, no todo es atribución estatal. Las comunidades autónomas tienen competencia en materia de juego privado (casinos, bingos y máquinas tragaperras) y también en lo que se refiere a las apuestas realizadas con máquinas. Dichas máquinas de apuestas se ubican en los salones de juego — cuyo número ha crecido espectacularmente en los últimos dos años-, así como en bares, dependiendo de las regulaciones establecidas por cada comunidad autónoma, de forma que a día de hoy ya es un hecho encontrar máquinas de apuestas al lado de las tragaperras en los establecimientos de hostelería. Se repite la historia de 1981 cuando el sector pilló con el pie cambiado al gobierno de la UCD. La diferencia es que ahora ya se conocen los efectos negativos que tiene el ubicar estos juegos en los bares y hace tiempo que se viene reclamando desde los ámbitos científicos y sociales las necesarias medidas regulatorias de limitación de acceso a los mismos, como medida de prevención de la adicción al juego.

La situación es tan grave, que en menos de tres años desde la legalización del juego online, éste ya es la segunda causa de adicción al juego para los pacientes que buscan 
asistencia por su problema de ludopatía —sólo por detrás de las máquinas tragaperras-y la principal causa de la adicción al juego en jóvenes (Chóliz, 2015).

\section{¿Es posible prevenir sin controlar la exposición ambiental?}

Sin duda que existen factores personales de vulnerabilidad para la adicción al juego pero, al igual que ocurre con el alcohol, son los ambientales los que en mayor medida favorecen y desencadenan la aparición y desarrollo de esta patología. El juego está bien visto socialmente, coincide con muchos de los valores prevalentes en nuestra sociedad sobre la obtención de dinero fácil y hasta es un ejemplo de actividad especulativa, que es la forma en que la economía de mercado obtiene sus mayores beneficios monetarios. Además, desde la legalización del juego online, la presencia en los medios de comunicación a través de publicidad y estrategias comerciales ha crecido de forma exponencial. Según datos del propio Ministerio de Hacienda, en 2015 las empresas del sector del juego online gastaron en publicidad y promoción 164 millones de euros (DGOJ, 2016b), que es más del doble de lo que aportaron en impuestos sobre el juego a Hacienda. Esto hace que, a día de hoy, el juego online sea omnipresente en los medios de comunicación de masas puesto que, para más inri, no existe una ley que regule específicamente la publicidad y las estrategias comerciales del juego de azar. Así pues, el problema no es que no haya campañas de prevención de adicción al juego, o que exista una concepción benévola sobre dicha actividad, sino que se está promocionando e incentivando de manera irresponsable.

Resulta sorprendente que casi cuatro años después de la concesión de las licencias para operar, todavía no haya un reglamento que ordene específicamente la publicidad y las técnicas comerciales del juego. La Administración ha dejado en manos del sector su propia regulación de la publicidad mediante un código de autocontrol, en lugar de establecer medidas concretas como las que se han tomado en tabaco y alcohol. Dicho código de conducta sobre las comunicaciones comerciales denominado Autocontrol resulta a todas luces insuficiente, ya que no tiene en cuenta las características especiales de la publicidad del juego, ni distingue entre comunicación comercial e incitación al consumo disfuncional. Además, ni siquiera lo han suscrito todas las empresas del sector.

No podemos olvidar que es el Estado quien, en su doble función de regulador del juego y garante de la seguridad y bienestar de los ciudadanos, debe cumplir con la obligación de definir claramente los límites de la publicidad y señalar con precisión las normas bajo las cuales debe publicitarse una actividad que, como se ha demostrado científicamente, es potencialmente adictiva y la causa de una grave patología psicológica de consecuencias a menudo muy graves. Entretanto, sin embargo, no solamente no se previenen los efectos adictivos del juego de azar, sino que esta actividad se promociona e incentiva, buscando en los jóvenes nuevos nichos de mercado.

\section{¿Y se puede prevenir el juego patológico sin legislar?}

En uno de los informes más exhaustivos y rigurosos sobre la prevención del juego excesivo realizado por investigadores del Alberta Gambling Research Institute (Williams, West y Simpson 2012) se concluye que la única forma realmente eficaz de prevenir el juego patológico es mediante políticas de juego, es decir, mediante la regulación. En este mismo informe se indica que otro tipo de medidas, incluso las educativas, si bien tienen su grado de utilidad, no son eficaces sin una adecuada normativa que legisle la disponibilidad y accesibilidad de los juegos de azar.

En este sentido, no es menos relevante el hecho de que la regulación del juego haya pasado de depender del Ministerio del Interior al de Hacienda. Este hecho, por intrascendente que parezca, es a nuestro juicio fundamental para entender la impermeabilidad que a veces se produce por parte de las autoridades sobre los riesgos de una actividad económica que están obligadas no sólo a fiscalizar, sino también a regular. Y la regulación no acaba con la legalización del juego; eso es sólo el principio, al menos en lo que se refiere a la prevención de la adicción al juego.

Finalmente, esta necesidad de legislar adecuadamente para la prevención de la adicción al juego no solamente se recoge y se defiende desde los ámbitos clínicos y sociales, sino que también viene requerida en el Informe del Parlamento Europeo sobre juego online en el mercado interior (Fox, 2013). En este informe se indica claramente que el juego "no es una actividad económica convencional debido a sus posibles efectos sociales negativos, en particular la ludopatía, con consecuencias y costes difíciles de estimar", que "debido a la naturaleza especial de los juegos de azar en línea, la protección de la salud humana y de los consumidores debe ser el principio rector fundamental al elaborar recomendaciones a nivel de la UE y legislación a nivel nacional' y que “... el Tribunal de Justicia ha confirmado que la oferta de juegos de azar es una actividad económica de naturaleza especial en la que se pueden justificar restricciones por razones imperiosas del interés general tales como la salud pública”. En lo que se refiere a la publicidad, tema que hemos comentado en el apartado anterior, "reitera su posición de que, en una materia tan delicada como el juego, la autorregulación del sector puede complementar a las normativas nacionales, pero nunca sustituirlas".

En conclusión, lo que no puede perderse de vista es que el juego no es una actividad económica convencional, sino que los beneficios empresariales de las empresas del juego proceden directamente de lo que pierden los jugadores cuando apuestan. Si tenemos en cuenta que quienes más 
juegan —y más pierden- son los jugadores patológicos, que son personas que padecen un trastorno mental a causa del juego, la regulación de esta actividad debe someterse a medidas especiales que prevengan en la medida de lo posible la aparición de una de las enfermedades mentales que causa mayor infelicidad a los pacientes y a sus familias. Por todo ello, es necesario que de una vez por todas se legisle desde las diferentes administraciones del Estado (estatal y autonómica), entendiendo que el juego, al igual que el alcohol o el tabaco, es una actividad económica especial que tiene serios riesgos para la salud que deben ser prevenidos mediante las adecuadas políticas de juego que se basen en las evidencias que proporciona la ciencia.

\section{Referencias}

APA (2013). Manual Diagnóstico y Estadístico de los Trastornos Mentales, DSM-5. Madrid: Editorial Médica Panamericana.

Chóliz, M. (2015). The challenge of online gambling: the effect of legalization on the increase in online gambling addiction. Journal of Gambling Studies, 1-8. doi: 10.1007/ s10899-015-9558-6.

Chóliz, M. y Sáiz, J. (2016). Regular el juego para prevenir la adicción: hoy más necesario que nunca. Adicciones, 28, 174-181.

DGOJ (2016a). Mercado del juego online en España. Informe trimestral, T1 2016. Dirección General de Ordenación de Juego. Madrid: Ministerio de Hacienda y Administraciones Públicas. Recuperado de la web: http://www. ordenacionjuego.es/es/estudios-informe.

DGOJ (2016b). Análisis Global del Mercado Nacional del Juego Online. Informe $4^{\circ}$ trimestre 2015. Dirección General de Ordenación del Juego. Madrid: Ministerio de Hacienda y Administraciones Públicas. Recuperado de la web: http://www.ordenacionjuego.es/es/estudios-informes.

Fox, A. (2013). Informe del Parlamento Europeo sobre juego online en el mercado interior. Documento de sesión PE506.088v02-00. Comisión del Mercado Interior y Protección del Consumidor, Parlamento Europeo.

Rodríguez-Martos, A. (2007). ¿Por qué es tan difícil legislar sobre el alcohol en España?. Adicciones, 19, 325-332.

Williams, R.J., West, B. y Simpson, R. (2012). Prevention of Problem Gambling. Informe preparado para Ontario Problem Gambling Research Centre y Ontario Ministry of Health and Long Term Care. 